\title{
On the Production of Severely Deformed Workpieces in Large Scales: A Step Towards Industrialization
}

\author{
E. BAGHERPOUR $\left(\mathbb{D},{ }^{1,5} \mathrm{~S}\right.$. MORTEZAEI,${ }^{2}$ R. EBRAHIMI, ${ }^{2}$ \\ H. MIYAMOTO ${ }^{3}$ and F. QODS ${ }^{4}$
}

1.-BCAST, Brunel University London, Uxbridge UB8 3PH, UK. 2.-Department of Materials Science and Engineering, School of Engineering, Shiraz University, Shiraz, Iran. 3.-Department of Mechanical Engineering, Doshisha University, Kyotanabe, Kyoto 610-0394, Japan. 4.-Faculty of Metallurgical and Materials Engineering, Semnan University, Semnan, Iran. 5.-e-mail: Ebad.Bagherpour@brunel.ac.uk

Improvement of the functional properties of aluminium and its alloys by grain refinement is an effective way to increase their applications. The capability of severe plastic deformation methods to produce ultrafine-grained materials has been well established. However, their industrial application is limited because of the required additional equipment and limitation of the product size. Due to the direct extrusion characteristic of the simple shear extrusion (SSE) method and consequently the minimal additional tools and expenses, SSE is a good candidate for commercialization. The aim of this research is to scale up the SSE products to facilitate their potential use in practical applications. To overcome the limitation on the length of the plunger and reduce the load of the SSE process, a design is proposed in the current research. Microstructural investigations and mechanical tests of commercial pure aluminium (AA1050) workpieces confirm the effectiveness of the proposed design on the grain refinement and its capability to reduce the processing load.

\section{INTRODUCTION}

The most used non-ferrous materials in industrial applications are aluminium and its alloys because of several advantages such as the high level of natural resources, low density, acceptable mechanical properties, appropriate corrosion resistance, excellent electrical conductivity, and relatively low cost. ${ }^{1}$ Improvement of mechanical properties of aluminium alloys is a key factor in their use in automotive and aerospace applications. It is extensively documented that the grain refinement of aluminium and its alloys is an influential approach to simultaneously improve their strength and plasticity (the details can be found in Ref. 2-4). Over 30 years of research in the various fields of severe plastic deformation (SPD) confirms the exceptional mechanical and physical properties of the ultrafinegrained (UFG) \nano-structured (NS) materials. ${ }^{5-8}$ The approach to the application of SPD methods in microstructural refinement is based on the heavy straining by imposing high pressures. The principle of SPD processing and the detailed mechanisms involved in the corresponding grain refinement are presented in several review articles and book chapters. ${ }^{9-13}$ As a step forward, much attention has been paid to the commercialization of SPD processing in recent years. ${ }^{14-16}$ Nevertheless, there are still few reports of successful application of SPD methods in industrial applications. ${ }^{17-19}$ To achieve the goal, several challenges must be resolved.

3The first challenge is the feasibility of SPD processing on the different types of materials including metals, alloys, and composites. Currently, extensive studies are being conducted to widen the range of materials that can be processed by SPD methods. Since the grain refinement by SPD processing needs repetition of the process, the second challenge is the possibility of continuous production of UFG materials by SPD processing, which is a great advantage for the reduction of production costs. Most of the efforts to overcome this problem have been concentrated on equal channel angular pressing (ECAP) $)^{20}$ as one of the most popular SPD methods. As will be explained later, despite the high potential of ECAP, some 
other methods having the same potential, but with higher performance, have been less considered. The last challenge is to scale up the SPD products. ${ }^{21}$ The main problems based on this are the nature of the method, tool design, and overcoming the friction. For instance, despite the great advantage of high-pressure torsion (HPT) ${ }^{22,23}$ in grain refinement, there are several restrictions on the product size. Regarding all the barriers to the industrialization of SPD processing, it can be concluded that reducing or removing additional equipment, making modifications to increase the production rate, and scaling-up of the SPD products are the most valuable key factors.

So far, more than 100 SPD methods invented ${ }^{24}$ some are independent, while others are related to another method or combination of two different methods. Additional equipment and/or a new design of the production lines in the methods based on ECAP, such as conshearing ${ }^{25}$ and ECAP-Conform, ${ }^{26}$ is needed. Furthermore, expensive devices are needed for the methods based on the application of torsion/shear under high pressure, such as HPT, rotation torsion, ${ }^{27}$ high-pressure shearing (tHPS), ${ }^{28}$ and continuous high-pressure torsion for bars/rods. ${ }^{29,30}$ Therefore, application of the methods based on direct extrusion for industrial uses is more probable because of the easy installation of the tools in a production line, their continuous nature, and consequently the low production cost. Among the different methods including twist extrusion (TE), ${ }^{31,32}$ simple shear extrusion (SSE), ${ }^{33-38}$ also named planar twist extrusion, ${ }^{39}$ pure shear extrusion, ${ }^{40}$ spread extrusion, ${ }^{41}$ and nonlinear rotary extrusion (NRE) ${ }^{42}$ only TE and SSE have been studied widely. Different deformation zones and shear planes of the TE and SSE process result in different strain distributions in the products, which, in turn, result in different properties. The maximum amount of strain is observed at the centre of the SS-extruded samples and is observed at the periphery of the T-extruded specimens. Furthermore, the microstructure and mechanical properties of the SS-extruded workpieces are more uniform through a section of the product. ${ }^{33-35}$ Also, the exceptional combination of high strength and ductility of the specimens after multi-passes of SSE processing ${ }^{35,36}$ and the ability to deform "hard to deform materials," such as Mg alloys ${ }^{43,44}$ and TWIP steels, ${ }^{45,46}$ make this process a good candidate for industrialization.

This study offers an approach to SSE processing to produce large samples, without any limitation on the product size. The proposed design is a powerful tool not only for SSE processing, but also for the other methods based on direct extrusion, such as TE.

\section{DESIGN CONCEPT}

To overcome the specimen's size limitation in SSE processing, it is essential to study the conventional die and tool design to find its limitations and shortcomings. In this section, the concepts of the conventional design, its restrictions, and its weaknesses are described in detail. After that, the concepts of a design that could overcome these limitations are defined in a way that can be used for future potential industrial applications, in which the fabrication of large ultrafine-grained specimens is indispensable.

\section{Conventional Die Design}

As shown in Fig. 1a, during SSE processing, the material tolerates gradually increasing simple shear strain without changing the cross-sectional area. At the middle of the deformation channel where maximum distortion occurs, the shear direction reverses. Afterward, the imposed shear strain gradually decreases to zero at the exit channel; consequently, the cross section of the specimen returns to its initial square cross section. In the conventional designs, a plunger similar to that in Fig. $1 \mathrm{~b}$ is used for processing. Although this type of design is simple, easy to construct and install, and cheap, it has two major weaknesses: the limitation in the length of the products and the inappropriate effect of friction on the process load. The limitations of the conventional design will be explained in this section.

\section{Limitation of the Length of the Workpieces}

The first and the most significant weakness of the design is the limitation of the length of the plunger, which, in turn, results in the limitation of the length of the work pieces and products. It is legitimate to consider the plunger as a vertical beam subjected to an axial load. Therefore, according to Euler's formula ${ }^{47}$ the maximum load (critical load) that a column can bear while staying straight can be defined by

$$
F_{\text {cr }}=\frac{\pi^{2} E I}{L_{\mathrm{p}}^{2}}
$$

where $E$ is the elastic modules, $I$ is the minimum area moment of inertia (second moment of area) of the cross section of the plunger, and $L_{\mathrm{p}}$ is the unsupported length of the plunger. For a rectangular plunger with a side length of $a$ (see Fig. 1b), $I$ is calculated by

$$
I=\frac{a^{4}}{12}
$$

Inserting Eq. 2 into Eq. 1, $P_{\text {cr }}$ will be:

$$
F_{\text {cr }}=\frac{\pi^{2} E a^{4}}{12 L_{\mathrm{p}}^{2}} \text {. }
$$




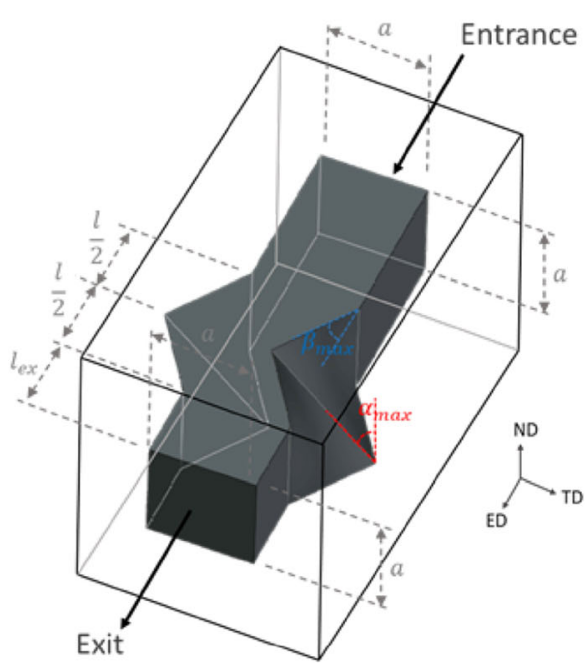

(a)
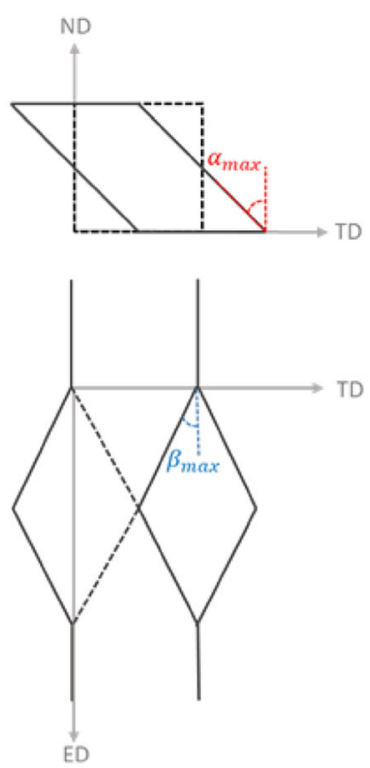

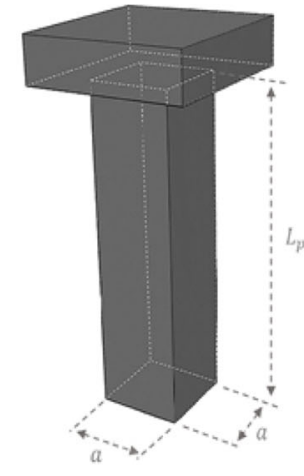

(b)

Fig. 1. Schematic presentation of (a) the SSE channel (3D, front and top views) and (b) a conventional plunger (punch) using in conventional dies.

The plunger will fail by buckling if the extrusion force $\left(F_{\text {ext }}\right)$ exceeds $F_{\text {cr }}$. Therefore, the consistency of the plunger is satisfied by

$$
F_{\text {ext }}<\frac{\pi^{2} E a^{4}}{12 L_{\mathrm{p}}^{2}}
$$

Hence, to protect the plunger from buckling, the length of the plunger must be in the below range:

$$
L_{\mathrm{p}}<\frac{\pi a^{2}}{2} \sqrt{\frac{E}{3 P_{\mathrm{ext}}}} .
$$

This means that the maximum length of the plunger can be processed by SSE using the conventional design $\left|\frac{\pi a^{2}}{2} \sqrt{\frac{E}{3 P_{\text {ext }}}}\right|$. As discussed in the next section ("Design to Overcome Specimen's Size Limitation" section), the extrusion pressure increases by increasing the sample length. Therefore, Eq. 5 may not be satisfied by increasing the length of the samples, which means the failure of the plunger. It is an important challenge in the processing of large samples because of the obvious requirement of a longer plunger for the longer samples, which could not be achieved in the conventional designs.

\section{Effect of Friction}

Bagherpour et al. ${ }^{48}$ proposed an analytical solution for the prediction of the process load based on an upper-bound model. They showed that the relative extrusion pressure for the SSE process is

$$
\begin{aligned}
\frac{P_{\text {ext }}}{\sigma_{f}}= & \frac{2}{\sqrt{3}}\left\{\frac{\tan \alpha_{\max }}{\alpha_{\max } \beta_{\max }} \ln \left(\tan \alpha_{\max }+\frac{1}{\cos \alpha_{\max }}\right)\right. \\
& \ln \left(\tan \beta_{\max }+\frac{1}{\cos \beta_{\max }}\right)+\tan \beta_{\max } \\
& +\frac{\operatorname{mtan} \alpha_{\max }}{\alpha_{\max } \tan \beta_{\max }} \ln \left(\tan \alpha_{\max }+\frac{1}{\cos \alpha_{\max }}\right) \\
& +\frac{\operatorname{mtan} \alpha_{\max } \tan \beta_{\max }}{3 \alpha_{\max }} \ln \left(\tan \alpha_{\max }+\frac{1}{\cos \alpha_{\max }}\right) \\
& \left.+\frac{\operatorname{mtan} \alpha_{\max }}{\sin \beta_{\max } \cos \beta_{\max }}+\frac{2 m}{a}\left(L_{\mathrm{w}}+l_{\mathrm{ex}}\right)\right\}
\end{aligned}
$$

where $L_{\mathrm{w}}$ is the length of the workpiece, $l_{\mathrm{ex}}$ is the length of the exit channel of the die, and $m$ is the constant friction factor. Since the applied strain is defined by $\alpha_{\max }$, its amount is assumed to be determined. Also, for a specific material the amount of $m$ is fixed. Therefore, the variable $\beta_{\max } \cdot \beta_{\max }$ relates to $\alpha_{\max }$ by ${ }^{48}$

$$
\tan \beta_{\max }=\frac{a \tan \alpha_{\max }}{L}
$$

where $L$ is the length of the deformation channel. For the designer, there are two different concepts in the design of the tools and die for SSE regarding the selection of $\beta_{\max }$. One may assume $L$ as a constant, which results in the increase of $\beta_{\max }$ by increasing the size of the workpiece. It was shown that for a defined $\alpha_{\max }$ there is a unique amount for the $\beta_{\max }$ $\left(\beta_{\max }^{\text {opt }}\right)$ by which the required extrusion pressure is 

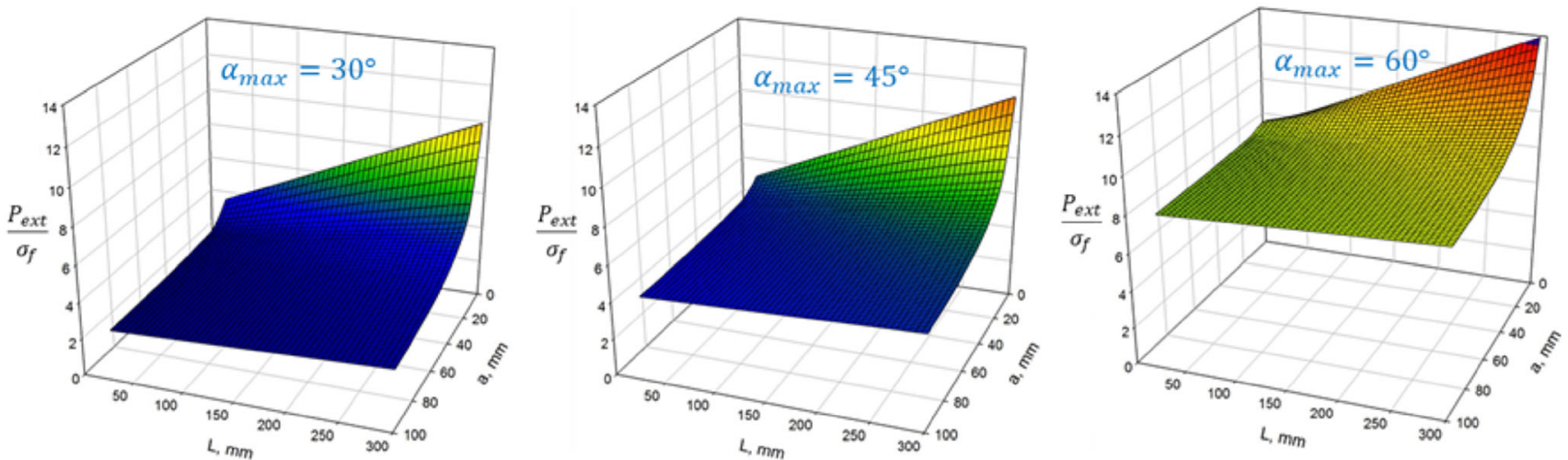

(a)

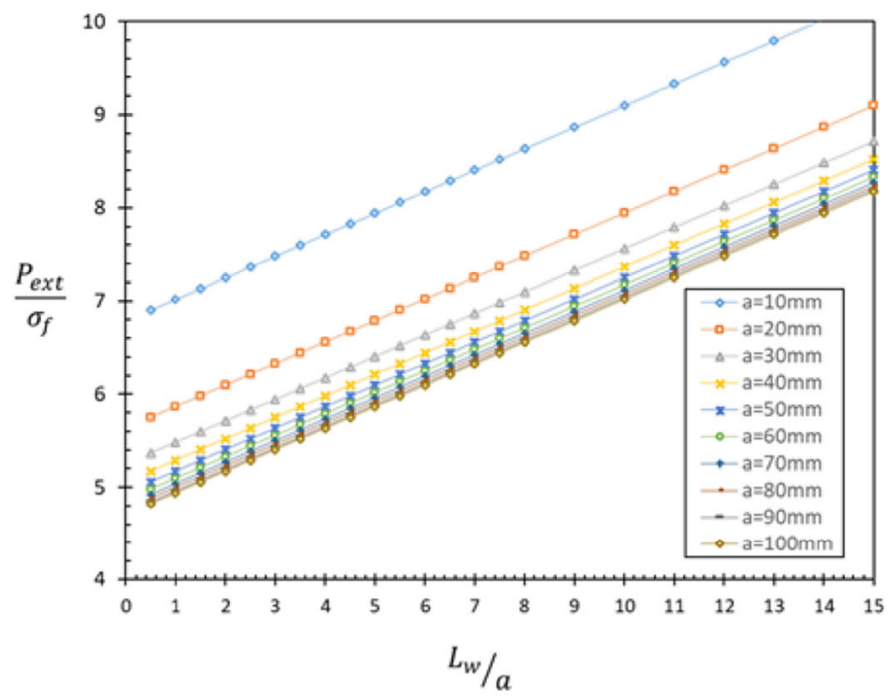

(b)

Fig. 2. Variation of the relative extrusion pressure with (a) increasing the size of the workpieces and the distortion angle for the FIC design and (b) the sample aspect ratio for the maximum distortion angle of $45^{\circ}$ and various side lengths for the MIC design.

minimized. ${ }^{48}$ Therefore, it is more efficient to assume $\beta_{\max }^{\text {opt }}$ as a fixed amount for $\beta_{\max }$ and select $L$ for different side lengths $(\alpha)$ using Eq. 6 . Hence, considering the fixed amount for $\alpha_{\max }$ and $\beta_{\max }$, the deformation length increases with increasing $a$. Therefore, it is valid to use the second approach in the rest of the current work. Consequently, Eq. 6 can be rewritten as

$$
\begin{aligned}
& \frac{P_{\text {ext }}}{\sigma_{f}}=\frac{2}{\sqrt{3}}\left\{\frac{\tan \alpha_{\max }}{\alpha_{\max } \beta_{\max }^{\text {opt }}} \ln \left(\tan \alpha_{\max }+\frac{1}{\cos \alpha_{\max }}\right)\right. \\
& \ln \left(\tan \beta_{\max }^{\mathrm{opt}}+\frac{1}{\cos \beta_{\max }^{\mathrm{opt}}}\right)+\tan \beta_{\max }^{\mathrm{opt}} \\
& \frac{\operatorname{mtan} \alpha_{\max }}{\alpha_{\max } \tan \beta_{\max }^{\text {opt }}} \ln \left(\tan \alpha_{\max }+\frac{1}{\cos \alpha_{\max }}\right) \\
& +\frac{m \tan \alpha_{\max } \tan \beta_{\text {max }}^{\mathrm{opt}}}{3 \alpha_{\max }} \ln \left(\tan \alpha_{\max }+\frac{1}{\cos \alpha_{\max }}\right) \\
& \left.+\frac{m \tan \alpha_{\max }}{\sin \beta_{\max }^{\text {opt }} \cos \beta_{\max }^{\text {opt }}}\right\}+\frac{4\left(L_{\mathrm{w}}+l_{\mathrm{ex}}\right) m}{\sqrt{3} a}
\end{aligned}
$$

Figure $2 \mathrm{a}$ shows the variation of relative extrusion pressure by increasing the size of the workpieces for three selected maximum distortion angles. The trends for all of the maximum distortion angles are the same. For a defined $a$, the extrusion pressure increases by increasing $L$. At first glance, it seems unusual that increasing the size of the samples has two contradictory effects. The extrusion pressure increases by increasing the length of the samples and decreasing its side length. However, deeper investigation of Fig. 2a by plotting the variation of relative extrusion pressure by changing the aspect ratio $\left(L_{\mathrm{w}} / a\right)$ can clarify the ostensible contradiction. Figure $2 \mathrm{~b}$ shows the variation of the relative extrusion pressure for the maximum distortion angle of $45^{\circ}$ for various side lengths. As seen, for a selected side length, the extrusion pressure depends linearly on the aspect ratio of the workpieces. However, for an aspect ratio, a lower extrusion process is achieved in a workpiece with a higher side length. This confirms the great significance of friction on the process load. Therefore, to 
increase the possibility of industrialization, methods and designs that can decrease the friction are of great importance.

\section{Design to Overcome a Specimen's Size Limi- tation}

In the previous section, the shortcomings of the conventional die design were discussed exhaustively. To overcome the shortcomings, the new design for an SSE die called the "movable inlet channel (MIC)" die was introduced in Ref. 48 by the current authors. In that publication, only the main concept of the design without any details was reported. The aim of the current section is to describe the details of the design to facilitate its repetition. Figure 3 shows a schematic representation of a special SSE design die with a movable inlet channel (MIC die). It can be seen that two of the four die walls are made by a specially designed plunger. Therefore, there is no relative motion between the work piece and die surfaces made by the plunger (shown by the patterned surfaces in Fig. 3).

As described in "Conventional Die Design" section, in the conventional design, there is an upper limit [Eq. 5] to the length of the plunger above which the plunger tends to buckle. As seen in Fig. 3, in an FIC design, the plunger consist of two

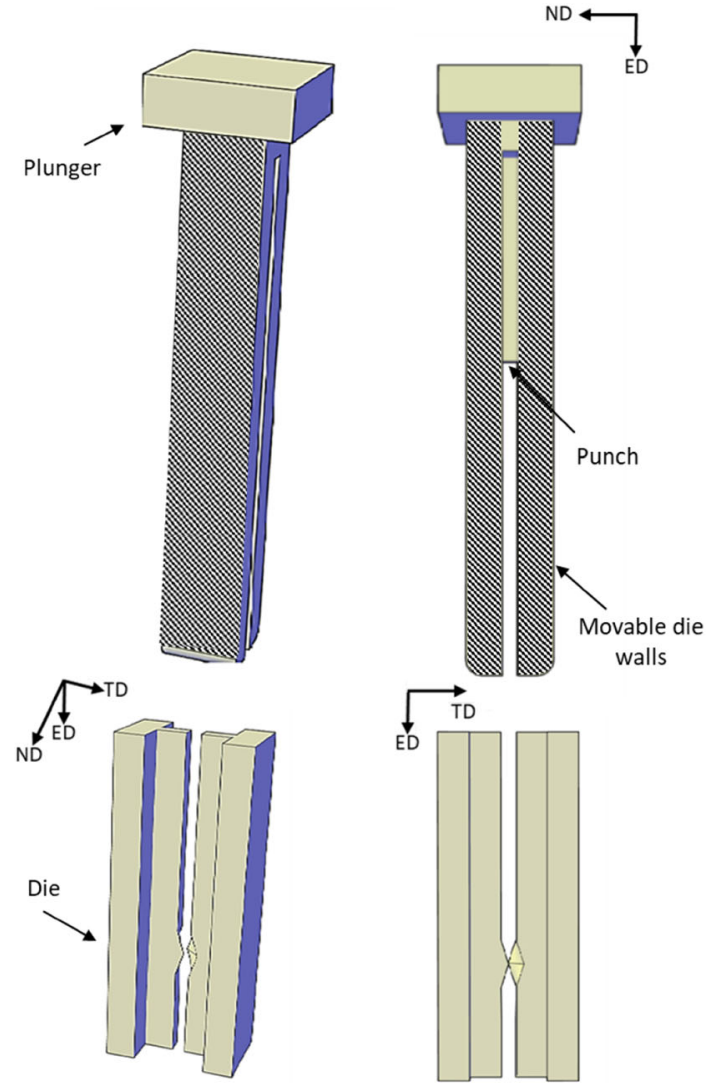

Fig. 3. Schematic representation of a special design of an SSE die with a movable inlet channel (MIC die). different parts, the movable die walls and the punch. Since the punch part pushes the workpieces through the extrusion channel, the punch is the part that should tolerate the load of the process. Therefore, if a part is supposed to buckle, that part is the punch. However, the movable die wall applies enough support for the punch, which prevents the buckling. In other words, there are no limitations to the length of the punch in the MIC design. Therefore, it is possible to deform samples with a high aspect ratio $\left(L_{\mathrm{w}} / a\right)$, which is prevalent in many industrial applications.

Another advantage of the MIC design is a reduction in the frictional forces. Since there is no relative motion between the workpiece and movable channels, frictional forces corresponding to these surfaces have no effect on the extrusion pressure of the process. Therefore, the relative pressure of the

$$
\begin{aligned}
\frac{P_{\mathrm{ext}}}{\sigma_{f}}= & \frac{2}{\sqrt{3}}\left\{\frac{\tan \alpha_{\max }}{\alpha_{\max } \beta_{\max }^{\mathrm{opt}}} \ln \left(\tan \alpha_{\max }+\frac{1}{\cos \alpha_{\max }}\right)\right. \\
& \ln \left(\tan \beta_{\max }^{\mathrm{opt}}+\frac{1}{\cos \beta_{\max }^{\mathrm{opt}}}\right)+\tan \beta_{\max }^{\mathrm{opt}} \\
& +\frac{m \tan \alpha_{\max }}{\alpha_{\max } \tan \beta_{\max }^{\mathrm{opt}}} \ln \left(\tan \alpha_{\max }+\frac{1}{\cos \alpha_{\max }}\right) \\
& \left.\left.+\frac{\left.m \tan \alpha_{\max } \tan \beta_{\max }^{\mathrm{opt}} \ln \left(\tan \alpha_{\max }+\frac{1}{3 \alpha_{\max }}\right)\right\}}{\cos \alpha_{\max }}\right)\right\} \\
& +\frac{2\left(L_{\mathrm{w}}+l_{\mathrm{ex}}\right) m}{\sqrt{3} a}
\end{aligned}
$$

Figure $4 \mathrm{a}$ shows the variation of the relative extrusion pressure of the SSE using an MIC design by increasing the size of the workpieces for three selected maximum distortion angles. The trend for the variations is the same as that of the conventional design (see Fig. 2a). However, the value of the extrusion pressure is lower for the MIC design. Also, the dependence of the load on the geometry is lower in the MIC design. To clarify the differences, Fig. $4 \mathrm{~b}$ illustrates variation of the relative extrusion pressure of the MIC design from the conventional one by changing the aspect ratio. Figure 4a shows this variation of various side lengths in SSE processing using a maximum distortion angle of $45^{\circ}$. As seen, by increasing the aspect ratio the difference of the extrusion pressures between two designs increases. In other words, by increasing the length of the samples, the effect of the MIC design on the reduction of the extrusion pressure increases. This confirms the importance of the MIC design for industrial applications of the SSE process.

\section{EXPERIMENTAL PROCEDURE}

Two different splitting dies have $45^{\circ}$ maximum distortion angles, and a side length of $20 \mathrm{~mm} \times$ $20 \mathrm{~mm}$ was designed and constructed for SSE 

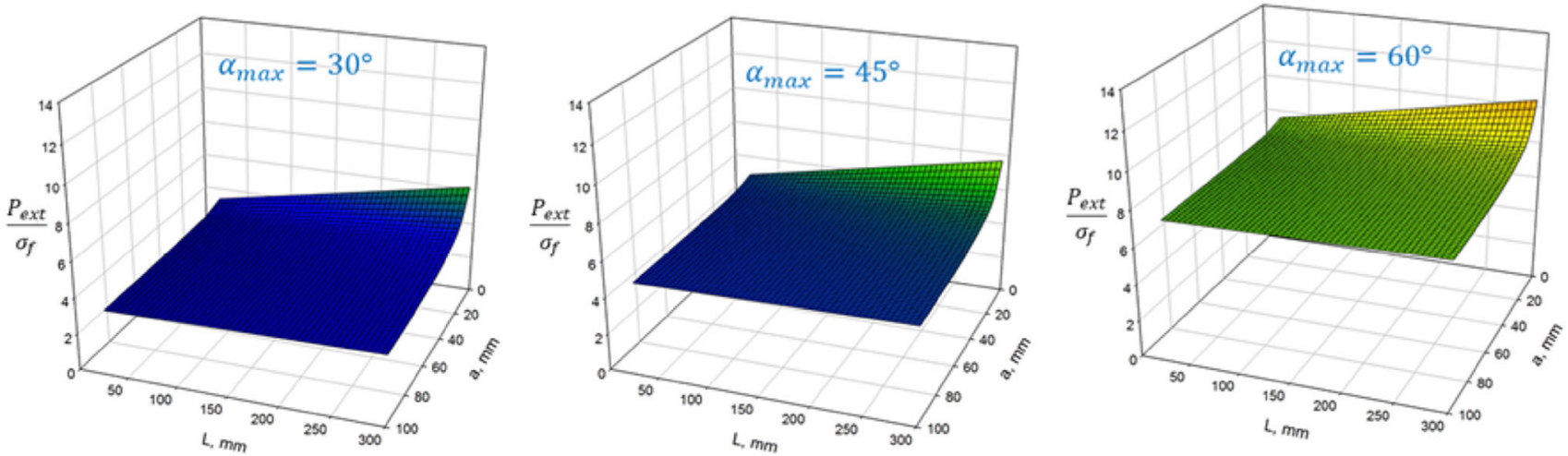

(a)

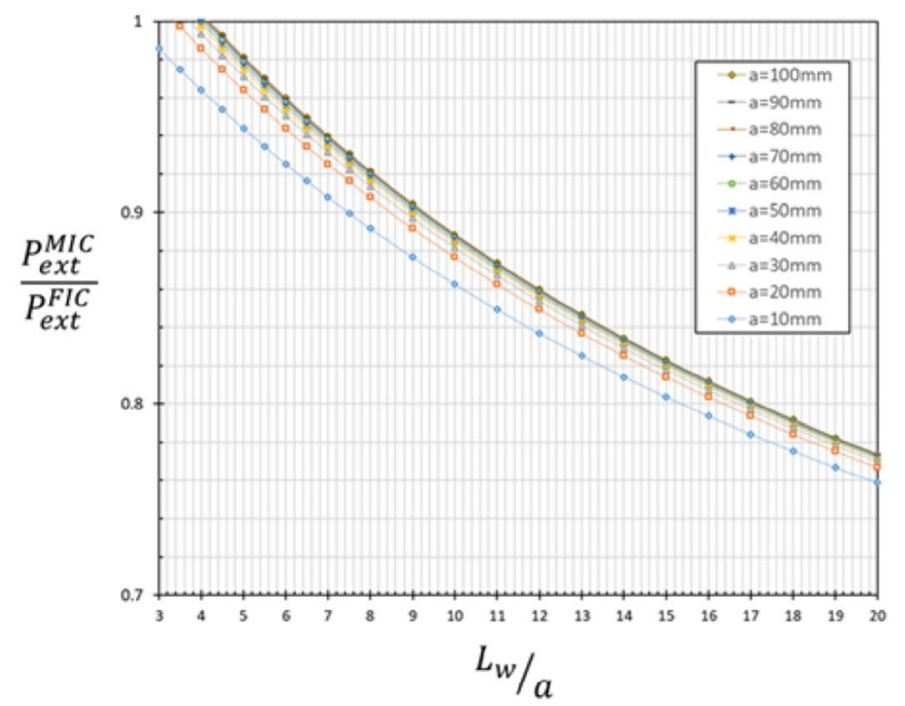

(b)

Fig. 4. Variation of the relative extrusion pressure with (a) increasing the size of the workpieces and the distortion angle for the MIC design and (b) the sample's aspect ratio for a maximum distortion angle of $45^{\circ}$ and various side lengths for the MIC design.

processing. One of the designs was based on the conventional design (FIC die). The FIC die was designed by an inclination angle of $22.2^{\circ}$, which is optimum. The other die was an MIC die with a $17.4^{\circ}$ inclination angle, shown in Fig. 5a. While the FIC die had the ability to deform the samples with a maximum length of $65 \mathrm{~mm}$, the MIC die was designed to deform samples with a maximum length of $160 \mathrm{~mm}$. Workpieces were deformed using a screw press with a controlled ram speed of $0.2 \mathrm{~mm} / \mathrm{s}$. To repeat the process, workpieces were rotated $90^{\circ}$ around the ED before the next pass. Commercial pure aluminium (AA1050) was used as the trial material.

Electron backscattering diffraction (EBSD) was used for microstructure investigations of the ED plane. After cutting the samples, grinding them, and polishing them using cloths and a suspension of $\mathrm{Al}_{2} \mathrm{O}_{3}$, the obtained surfaces were electrically polished in $20 \% \quad \mathrm{HClO}_{4}-80 \% \quad \mathrm{C}_{2} \mathrm{H}_{5} \mathrm{OH}$ using a DC voltage of $35 \mathrm{~V}$ for $20 \mathrm{~s}$ at $273 \mathrm{~K}$. EBSD observations were accomplished by a JEOL $7001 \mathrm{~F}$ scanning electron microscope (FE-SEM) equipped with a field emission gun operating at $20 \mathrm{kV}$. EBSD data were analysed by the INCA suite 4.09 software package. For the EBSD measurements of the samples after SSE, approximately 300 grains were used. Then, we used their average for the average grain size and reported the maximum and minimum measured values as superscripts and subscripts, respectively.

For transmission electron microscopy (TEM), specimens were cut from the centre of the deformed workpieces using a micro-cutter. The specimen surface was mechanically polished to $100 \mu \mathrm{m}$ thickness using abrasive papers before further thinning by a twin-jet polishing Tenupole 5 facility (Struers Co., Ltd.) with the same voltage, temperature, and solution as in EBSD sample preparation. Next, the specimens were polished by ion beams using a Gatan 691 precision ion polishing system (PIPS). For TEM observations, a transmission electron microscope (JEOL JEM-2100F) with acceleration voltage of $200 \mathrm{kV}$ was used. 


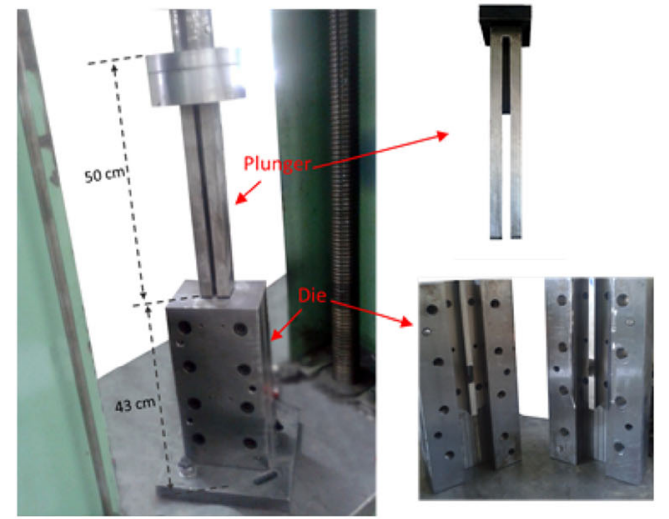

(a)

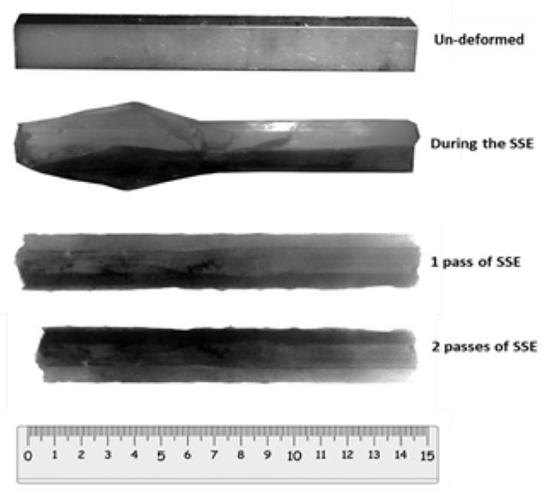

(b)

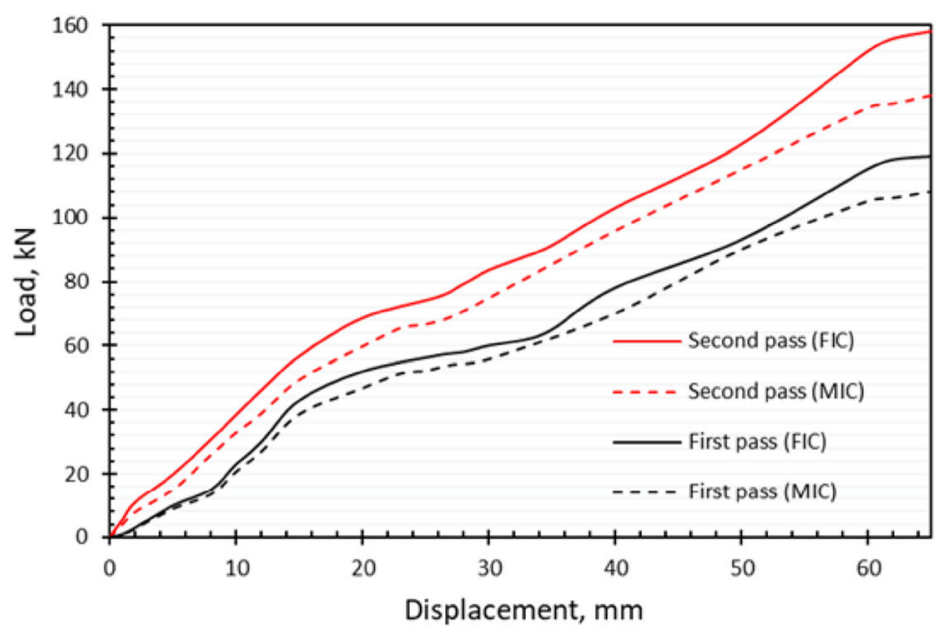

(c)

Fig. 5. (a) Used FIC die set (die + plunger) in the current research; (b) the initial sample and samples during and after SSE processing; (c) variation of the processing load with punch displacement for MIC and FIC dies.

To investigate the mechanical properties of the samples, both tensile and hardness tests were used. Vickers microhardness was evaluated by applying $100 \mathrm{~g}$ for $15 \mathrm{~s}$ dwell time on a polished surface. Samples for tensile tests were machined from the centre of the deformed workpieces with an orientation along the ED, $1 \mathrm{~mm}$ gage width, and $3 \mathrm{~mm}$ gage length. Tensile tests were accomplished at room temperature with a SHIMAZU AGS-10kND tensile testing machine operating at a nominal strain rate of $1.1 \times 10^{-3} \mathrm{~s}^{-1}$. To check the consistency of the results, five tensile tests were used on each sample condition.

\section{RESULTS AND DISCUSSION}

Figure $5 \mathrm{~b}$ shows the samples during SSE, after the first, and after the second pass of SSE. As seen, the deformation during the process is perfect and the samples successfully deformed up to two passes without any crack formation in the samples and/or any damage of the tools (die/plunger). Specimens completely filled up the die cavity, as can be observed from the sample extracted from the die during the process in Fig. 5b. The reason is that the channel was always filled up and the front specimen provided enough back-pressure for the rear one. Applying higher passes was not possible because of the $200 \mathrm{kN}$ capacity of the pressing machine. The processing load of the MIC die compares with the FIC die in Fig. 5c. To avoid the effect of the sample length, the loads for processing 6.5-mm-long samples for both MIC and FIC designs were compared. As seen, by using an MIC design the processing load decreases for both the first and second passes of SSE. This happens as a result of the lower frictional forces in the MIC design.

Figure 6a displays an inverse pole figure colour map and corresponding boundary map of the undeformed workpiece. An almost fully annealed grain microstructure had a grain size of about $240 \mu \mathrm{m}$ using the equivalent circle diameter is observed in the undeformed sample. More than $60 \%$ of the boundaries are high-angle grain boundaries (HAGBs). Figure $6 \mathrm{~b}$ and $\mathrm{c}$ illustrates the inverse pole figure EBSD orientation and corresponding boundary misorientation maps of the severely deformed samples after one and two passes of 
On the Production of Severely Deformed Workpieces in Large Scales: A Step Towards
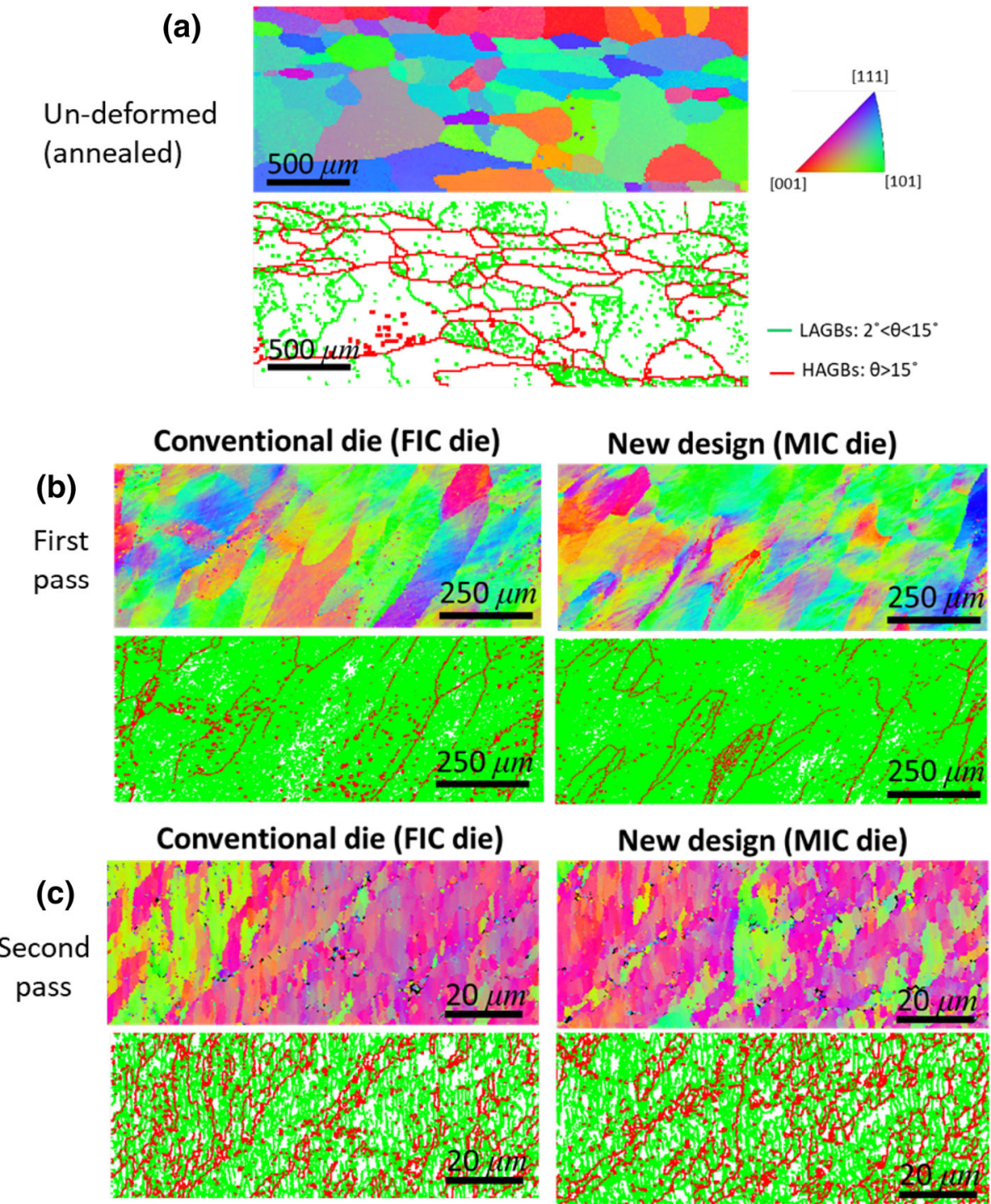

Fig. 6. Inverse pole figure EBSD orientation colour map and corresponding boundary misorientation map of (a) the un-deformed, (b) first-pass, and (c) second-pass workpieces deformed by MIC and FIC dies (note the difference in the scale bars) (Color figure online).

SSE, respectively. By increasing the number of passes, gradually some new high-angle grain boundaries appear. Therefore, the microstructure develops more finely and smoothly. The detailed microstructure features of the deformed samples are shown in Table I. As seen, for the FIC design, the mean misorientation angle changes from $\sim 7.5^{\circ}$ to $\sim 15.5^{\circ}$ from the first to the second pass. The same trend is seen in the MIC design, where the mean misorientation angle changes from $\sim 7.7^{\circ}$ to $\sim 15.1^{\circ}$ by increasing the number of passes from 1 to 2 . By increasing the number of pass, gradually some new high-angle grain boundaries appear. Hence, the microstructure gently becomes finer. The grain size decreases by increasing the number of passes in all the planes. The grain size reduces severely from $240 \mu \mathrm{m}$ at the initial stage to about $5.2 \mu \mathrm{m}$ and $1.5 \mu \mathrm{m}$ for the first and second passes of SSE with the FIC design, respectively. Approximately the same grain sizes $(5.4 \mu \mathrm{m}$ and $1.6 \mu \mathrm{m}$ for the first and the second passes, respectively) are observed for the MIC design. Therefore, for both the MIC and FIC design, the fraction of HAGBs and mean misorientation angle increase by repeating SSE. As a result of the change in the misorientation angle, the grain size decreases significantly after two passes of SSE. All of the measured EBSD microstructural features are approximately the same for the MIC and FIC designs, particularly if taking into account the parameters' ranges in Table I. This confirms the same flow behaviour of both designs. Small deviations between the parameters may have originated for three reasons: first, the sample preparation conditions, especially the region from which the sample is extracted, where a small change in place may result in a difference in 


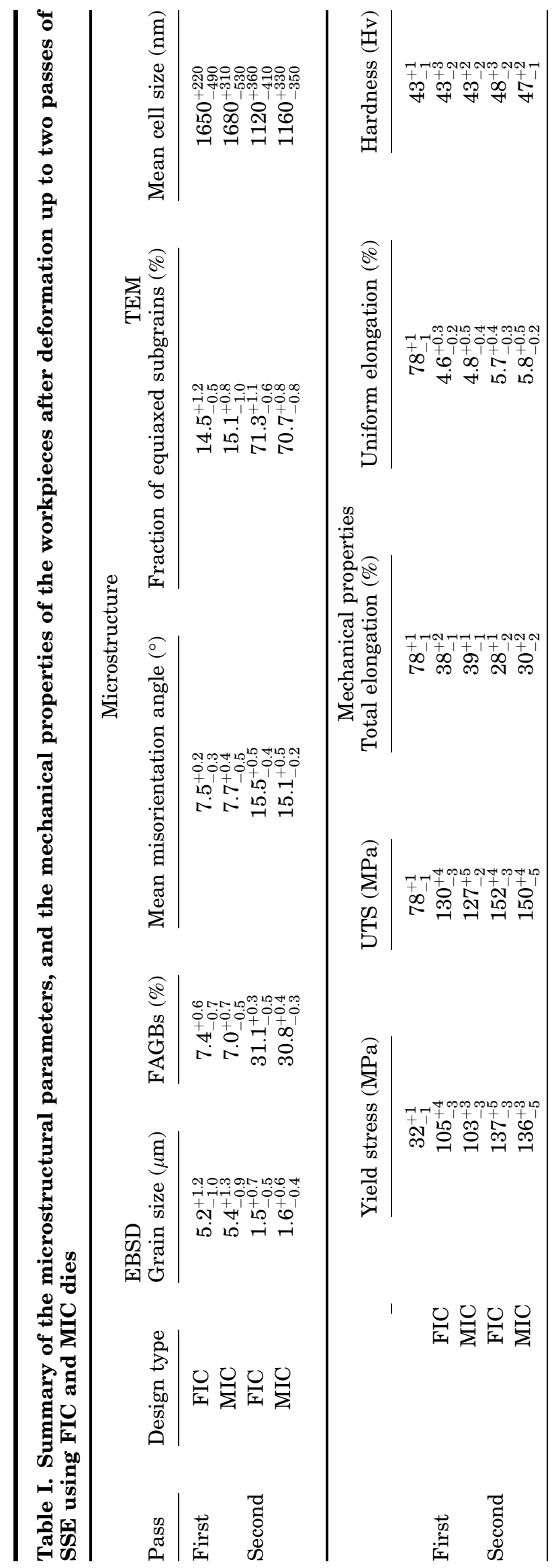



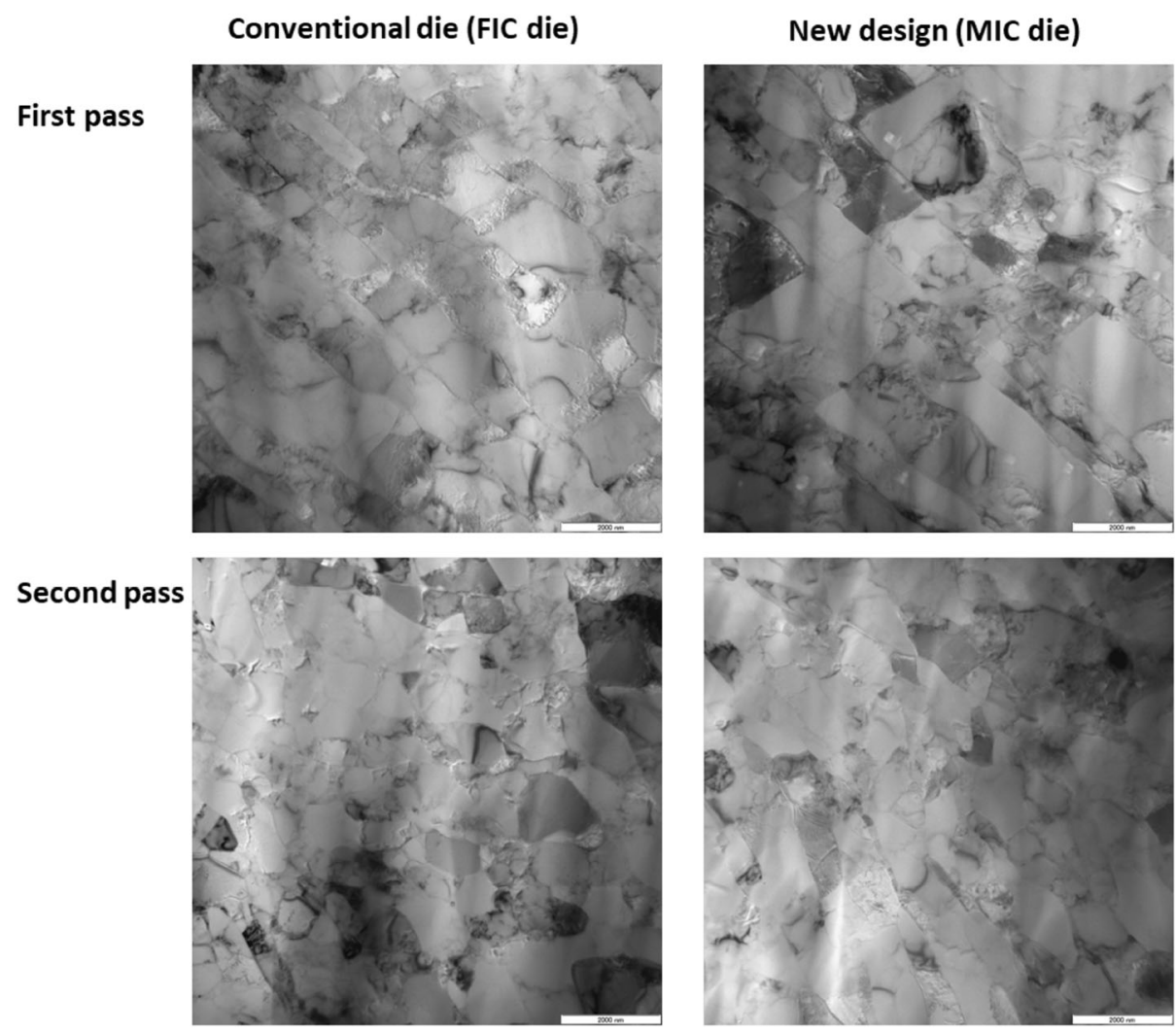

Fig. 7. TEM images of the samples after first- and second-pass SSE.

the microstructural features (refer to Refs. 33, 35, 49, and 50); second, from the different lengths of the deformation channels, which, in turn, result in different strain reversal behaviour. ${ }^{34,36,49}$

Figure 7 shows transmission electron micrographs of the deformed workpieces viewed from the ED plane and taken from the central region of the samples. For the samples deformed by FIC and MIC dies, the microstructure after the first pass of SSE consists of strongly elongated subgrains with mean lamellar boundary (LBs) spacing of $710_{-21}^{+23} \mathrm{~nm}$ and $706_{-18}^{+25} \mathrm{~nm}$ for the FIC and MIC designs, respectively. Almost $86 \%$ of the cells are elongated. Dislocation cell boundaries are observed inside the LBs. The TEM images of the second-pass workpieces and microstructure include both elongated and equiaxed subgrains. For the FIC sample approximately $71 \%$ of the investigated area is occupied by equiaxed subgrains. This area is about $65 \%$ of the MIC die. This difference may be attributed to the different lengths of the deformation channel in the different designs and consequently the different strain reversal behaviour ${ }^{33-35}$ of the design. However, it is not possible to reach a conclusion about the main reason for this difference in the current study and more detailed study, which could be the aim of separate research, is required. Nevertheless, considering the TEM parameters in Table I, the changes of the total behaviour of microstructural features are similar for the MIC and FIC designs. A more uniform microstructure with a higher fraction of equi-axed subgrains is achieved in a second pass for both the MIC and FIC designs.

A summary of the mechanical properties of the workpieces is shown in Table I. True stress- strain curves can be found in Supplementary Figure S1; refer to the online supplementary material. By increasing the number of passes, the hardness, yield strength, and UTS increased as a result of grain refinement. Because of the approximatly same microstructural changes of samples deformed with the MIC die as those deformed by the FIC die, the mechanical properties of the workpieces are the same as well. Compared with the undeformed workpieces, in workpieces deformed by two passes with the FIC die, the yield strength and UTS increase by $\sim 328 \%$ and $\sim 95 \%$, respectively, while the increase of these properties is $\sim 325 \%$ and $93 \%$ for the workpieces deformed by the MIC die. On the other hand, the elongation of the workpieces decreased significantly by increasing the plastic strain. The reduction of total and uniform elongation after two passes of SSE is $\sim 64 \%$ and $93 \%$, respectively, for the samples deformed in the FIC die. For the workpieces deformed in the MIC die, the decrease in the uniform elongation is approximately the same as for those deformed in the FIC die but the reduction in the total elongation in $3 \%$ lower. Generally, it can be concluded that the 
mechanical properties of the workpieces do not depend on the die design. The small difference in the mechanical properties $(<3 \%)$ attributed to the differences in the microstructural parameters was discussed previously.

\section{CONCLUSION}

Despite the many advantages of the severe plastic deformation methods, they have not found their way into industrial applications. Easy installation of the tools and the ability of the methods to deform large samples are the key factors in the commercialization of the SPD methods. Because SSE is a method based on direct extrusion, it can be easily installed. However, the application of this method in the production of large samples is a challenge. In this research, a design to overcome the specimen's size limitation and reduce the load of the process has been proposed for SSE in order to apply the method to large-scale production. Conventional designs are not suitable for the production of large samples because of the limitation of the length of the plunger, which results in limitation of the length of the sample as well as the effect of friction on the process load. The proposed design is a powerful tool not only for SSE processing, but also for the other methods based on direct extrusion, such as TE. The main achievements of this research are summarised below.

1. The plunger of the proposed design (MIC design) consists of two different parts, the movable die walls and the punch. Because there is enough support of the movable die wall for the punch to prevent buckling, there is no limitation of the length of the punch in the MIC design. In addition, there is no relative motion between the workpiece and the movable channels in the MIC design. Therefore, frictional forces corresponding to these surfaces have no effect on the extrusion pressure of the process. Consequently, the load of the process is lower in the MIC die than in the FIC design.

2. By increasing the specimen's aspect ratio the difference of the extrusion pressures between the two designs increases. In the other words, by increasing the length of the samples, the effect of the MIC design on the reduction of the extrusion pressure increases.

3. Experiments confirm the lower processing load of the MIC design as well as the effectiveness of the proposed design on grain refinement.

4. Tensile tests and hardness measurements confirm the same mechanical properties of the samples deformed by FIC and MIC dies.

5. The new MIC design can be successfully replaced by the conventional FIC design with approximately the same product performance, but with lower forces and costs and the ability to produce large workpieces.

\section{ACKNOWLEDGEMENTS}

The authors appreciate the financial support of Shiraz University for the use of the SSE facilities in the Department of Materials Science and Engineering, and the Metallic Materials Science Laboratory of Doshisha University for the application of electron microscopes (SEM/TEM).

\section{OPEN ACCESS}

This article is distributed under the terms of the Creative Commons Attribution 4.0 International License (http://creativecommons.org/licenses/by/4.0/), which permits unrestricted use, distribution, and reproduction in any medium, provided you give appropriate credit to the original author(s) and the source, provide a link to the Creative Commons license, and indicate if changes were made.

\section{ELECTRONIC SUPPLEMENTARY MATERIAL}

The online version of this article (https://doi.org/ 10.1007/s11837-019-03782-4) contains supplementary material, which is available to authorized users.

\section{REFERENCES}

1. I. Polmear, D. St. John, J.F. Nie, and M. Qian, Light Alloys: Metallurgy of the Light Metals, 5th ed. (Oxford: Butterworth-Heinemann/Elsevier Inc, 2017), pp. 1-107.

2. I. Sabirov, M.Y. Murashkin, and R.Z. Valiev, Mater. Sci. Eng., A 560, 1 (2013).

3. R.-G. Guan and D. Tie, Acta. Metall. Sin. Eng. L. 30, 409 (2017).

4. R.Z. Valiev, Y. Estrin, Z. Horita, T.G. Langdon, M.J. Zechetbauer, and Y.T. Zhu, JOM 58, 33 (2006).

5. I.A. Ovid'ko, R.Z. Valiev, and Y.T. Zhu, Prog. Mater Sci. 94, 462 (2018).

6. X. Sauvage, G. Wilde, S.V. Divinski, Z. Horita, and R.Z. Valiev, Mater. Sci. Eng., A 540, 1 (2012).

7. R.Z. Valiev, Mater. Trans. 55, 13 (2014).

8. R.Z. Valiev and T.G. Langdon, Adv. Eng. Mater. 12, 677 (2010).

9. R. Kocich, P. Lukáč, in Handbook of Mechanical Nanostructuring, vol. 1. (Wiley, 2015), p. 237.

10. A. Rosochowski and L. Olejnik, in Microstructure Evolution in Metal Forming Processes (Elsevier Ltd., 2012), p. 114.

11. R.Z. Valiev, in Nanostructured Metals and Alloys: Processing, Microstructure, Mechanical Properties and Applications (Elsevier Ltd., 2011), p. 3.

12. R.Z. Valiev, Y. Estrin, Z. Horita, T.G. Langdon, M.J. Zehetbauer, and Y. Zhu, JOM 68, 1216 (2016).

13. T. Sakai, A. Belyakov, R. Kaibyshev, H. Miura, and J.J. Jonas, Prog. Mater Sci. 60, 130 (2014).

14. T.C. Lowe, JOM 58, 28 (2006).

15. R.Z. Valiev, I.P. Semenova, V.V. Latysh, H. Rack, T.C. Lowe, J. Petruzelka, L. Dluhos, D. Hrusak, and J. Sochova, Adv. Eng. Mater. 10, B15 (2008).

16. T.C. Lowe and Y.T. Zhu, Adv. Eng. Mater. 5, 373-378 (2003).

17. L. Mishnaevsky, E. Levashov, R.Z. Valiev, J. Segurado, I. Sabirov, N. Enikeev, S. Prokoshkin, A.V. Solov'yov, A. Korotitskiy, E. Gutmanas, I. Gotman, E. Rabkin, S. Psakh'e, 
L. Dluhoš, M. Seefeldt, and A. Smolin, Mater. Sci. Eng., $R$ 81, 1 (2014).

18. I. Sabirov, N.A. Enikeev, M.Y. Murashkin, and R.Z. Valiev, Bulk Nanostructured Materials with Multifunctional Properties, ed. I. Sabirov, N.A. Enikeev, M.Y. Murashkin, and R.Z. Valiev (Cham: Springer International Publishing, 2015), pp. 101-113.

19. R.Z. Valiev, I. Sabirov, A.P. Zhilyaev, and T.G. Langdon, JOM 64, 1134 (2012).

20. R.Z. Valiev and T.G. Langdon, Prog. Mater Sci. 51, 881 (2006).

21. S. Ferrasse, V.M. Segal, F. Alford, J. Kardokus, and S. Strothers, Mater. Sci. Eng., A 493, 130 (2008).

22. K. Edalati and Z. Horita, Mater. Sci. Eng., A 652, 325 (2016).

23. A.P. Zhilyaev and T.G. Langdon, Prog. Mater Sci. 53, 893 (2008).

24. E. Bagherpour, N. Pardis, M. Reihanian, and R. Ebrahimi, Int. J. Adv. Manuf. Technol. 100, 1647 (2019).

25. H. Utsunomiya, K. Hatsuda, T. Sakai, and Y. Saito, Mater. Sci. Eng., A 372, 199 (2004).

26. C. Xu, S. Schroeder, P.B. Berbon, and T.G. Langdon, Acta Mater. 58, 1379 (2010).

27. M. Wang and A. Shan, J. Mater. Process. Technol. 202, 549 (2008).

28. J.T. Wang, Z. Li, J. Wang, and T.G. Langdon, Scr. Mater. 67, $810(2012)$

29. K. Edalati and Z. Horita, J. Mater. Sci. 45, 4578 (2010).

30. K. Edalati, S. Lee, and Z. Horita, J. Mater. Sci. 47, 473 (2012).

31. Y. Beygelzimer, D. Orlov, and V. Varyukhin, TMS Annual Meeting, ed. Y.T. Zhu, T.G. Langdon, R.S. Mishra, S.L. Setniatin, M.J. Saran, and T.C. Lowe (Hoboken: Wley, 2002), pp. 297-304.

32. Y. Beygelzimer, V. Varyukhin, S. Synkov, and D. Orlov, Mater. Sci. Eng., A 503, 14 (2009).

33. E. Bagherpour, F. Qods, R. Ebrahimi, and H. Miyamoto, Mater. Sci. Eng., A 674, 221 (2016).

34. E. Bagherpour, F. Qods, R. Ebrahimi, and H. Miyamoto, Mater. Sci. Eng., A 666, 324 (2016).

35. E. Bagherpour, F. Qods, R. Ebrahimi, and H. Miyamoto, Mater. Sci. Eng., A 679, 465 (2017).
36. E. Bagherpour, F. Qods, R. Ebrahimi, H. Miyamoto, in 6th International Biennial Conference on UltraFine Grained and Nanostructured Materials (UFGNSM 2017), ed. by M.H. Sohi and C. Zamani (American Institute of Physics Inc., vol. 1920, 2018).

37. N. Pardis and R. Ebrahimi, Mater. Sci. Eng., A 527, 355 (2009).

38. H. Sheikh, R. Ebrahimi, and E. Bagherpour, Mater. Des. 109, 289 (2016).

39. Y. Beygelzimer, D. Prilepo, R. Kulagin, V. Grishaev, O. Abramova, V. Varyukhin, and M. Kulakov, J. Mater. Process. Technol. 211, 522 (2011).

40. A. Eivani, Mater. Lett. 139, 15 (2015).

41. Y. Beygelzimer, V. Varyukhin, A. Reshetov, S. Synkov, D. Orlov, in TMS Annual Meeting (United States, 2006), p. 119.

42. E. Bagherpour, N. Komada, H. Fujiwara, H. Miyamoto, in The 66th Japanese Joint Conference for the Technology of Plasticity (Japan, 2015), p. 193.

43. N.B. Tork, N. Pardis, and R. Ebrahimi, Mater. Sci. Eng., A 560, 34 (2013).

44. N.B. Tork, H. Saghafian, S.H. Razavi, K.J. Al-Fadhalah, R. Ebrahimi, and R. Mahmudi, J. Mater. Res. Technol. 8, 1288 (2019).

45. E. Bagherpour, M. Reihanian, and R. Ebrahimi, Mater. Des. 40, 262 (2012).

46. E. Bagherpour, M. Reihanian, and R. Ebrahimi, Mater. Des. 36,391 (2012).

47. F. Beer, E.R. Johnston Jr. J. DeWolf, and D. Mazurek, Mechanics of Materials, 5th ed. (New York: McGraw-Hill Education, 2008), p. 606.

48. E. Bagherpour, R. Ebrahimi, and F. Qods, Mater. Des. 83, 368 (2015).

49. E. Bagherpour, F. Qods, R. Ebrahimi, and H. Miyamoto, Metals 8, 583 (2018).

50. E. Bagherpour, F. Qods, R. Ebrahimi, and H. Miyamoto, Mater. Trans. 57, 1386 (2016).

Publisher's Note Springer Nature remains neutral with regard to jurisdictional claims in published maps and institutional affiliations. 